\title{
Spaces of Continuous Sesquilinear Forms Associated with Unbounded Operator Algebras
}

\author{
K. SCHMÜDGEŃ
}

Sei $\mathcal{A}$ eine abgeschlossene * Algebra unbeschränkter Operatoren auf einem dichten invarianten Bereich $D$ eines Hilbert-Raumes und $\mathscr{L}_{\mathfrak{A}}\left(D, D^{\prime}\right)$ der Vektorraum aller stetigen Sequilinearformen auf $\mathcal{D} \times \mathcal{D}$ bezüglich der Graphtopologie von $\mathcal{A}$. Wir verallgemeinèn einige grundlegende Resultate aus der Theorie der von-Neumann-Algebren (das von-Neumannsche Bikommutantentheorem, das Kaplanskysche Dichtetheorem) auf gewisse lineare Unterräume von $\mathscr{L}_{\mathcal{U}}\left(\mathcal{D}, \mathcal{D}^{\prime}\right)$.

ІІусть $\mathcal{A}$ замкнутая *-алгебра неограниченных операторов заданных на плотноп инвариантной области $\mathcal{D}$ в некотором гильбертовом пространстве, и пусть $\mathscr{L} \mathfrak{d}\left(\mathcal{D}, \mathcal{D}^{\prime}\right)$ векторное пространство всех полуторалинейных форм на $\mathscr{D} \times \mathscr{D}$, непрершвных относительно топологии порождённой графиками операторов из $\mathcal{A}$. Мы обобщаем несколько основных результатов теории алгебр фон Неймана (теорема фоп Неймана о бикоммутанте, теорема Капланского о плотности) на некоторые линейные подпространства пространства $\mathscr{L} \mathcal{A}\left(\mathcal{D}, \mathcal{D}^{\prime}\right)$.

Let $\mathcal{A}$ be a closed *-algebra of unbounded operators on a dense invariant domain $\dot{D}$ of a Hilbert space, and let $\mathscr{I}_{\mathcal{A}}\left(\mathscr{D}, D^{\prime}\right)$ be the vector space of all continuous sequilinear forms on $D \times D$ relative to the graph topology of $\mathcal{A}$. We generalize some basic results of the von Neumann algebra theory (von Neumann bicommutant theorem, Kaplansky density theorem) to certain linear subspaces of $\mathscr{L}_{\mathcal{A}}\left(\mathscr{D}, \mathcal{D}^{\prime}\right)$.

\section{Introduction}

In this paper we prove some results which could be interpreted as generalizations of the two fundamental theorems in von Neumann algebra theory, the von Neumann bicommutant theorem and the Kaplansky density theorem, to certain vector spaces of continuous sesquilinear forms which are associated with unbounded operator algebras.'Precise definitions of these spaces will be given later.

The attempts to generalize the bicommutant theorem, for instance, to unbounded operator algebras meets serious difficulties in general. We shall illustrate this by a very simple example:-Let $A$, be the $*$-algebra of all polynomials in the multiplication operator by the independent variable $t$ on the dense domain $\mathcal{D}:=\left\{\varphi \in L^{2}(\mathbf{R}) ; t^{n} \varphi(t)\right.$ $\epsilon L^{2}(\mathbf{R})$. for all $\left.n \in \mathbf{N}\right\}$ of the Hilbert space $L^{2}(\mathbf{R})$. Then the strong-operator topology on $\mathcal{A}$ is equal to the finest locally convex topology on the vector space $\mathcal{A}$ (see e.g. [16]); so that $\mathcal{A}$ is closed in $L^{+}(\mathcal{D})$ with respect to the strong-operator topology. Since the bicommutant of $\mathcal{A}$ (in any reasonable definition) certainly contains all multiplication operators by bounded functions, $\mathcal{A}$ is different from its bicommutant.

$J_{n}$ order to get versions of the bicommutant theorem, there are (at least) two ways to overcome the difficulties met by the preceding example. The firstione is to replace the strong-operator topology by a weaker locally convex topology where we take only the seminorms $x \rightarrow\|x \varphi\|$ for certain "well-behaved" vectors $\varphi \in \mathcal{D}$. For instance, 
we could take all vectors $\varphi \in D$ for which the $O^{*}$-algebra $\mathcal{A} \uparrow \mathcal{A} \varphi$ is essentially selfadjoint on its domain $\mathcal{A} \varphi$. But for general $O^{*}$-algebras $\mathcal{A}$ on $\mathcal{D}$ it is not known if there exist such vectors $\varphi \in \mathscr{D}$ except, of course, zero. The second way is to enlarge the *-algebra $\mathcal{A}$. For instance, in the above example, a bicommutant theorem holds if we replace the $*$-algebra $\mathcal{A}$ by the $*$-algebra on $\mathcal{D}$ which is generated by the multiplication operators determined by the functions $t$ and $(t+\mathrm{i})^{-1}$.

One natural candidate for a generalization of the theory of-von Neumann algebras to the unbounded case is the class of $\mathrm{EW}^{*}$-algebras which were invented by Dixos [4] and studied also by INOUE [6]. EW*-algebras strongly resemble $W^{*}$-algebras, in a number of ways. But, in the author's opinion, this class is too restrictive for most of the interesting unbounded operator algebras. For instance, it is easy to see that there is no $O^{*}$-algebra $\mathcal{A}$ on $\mathscr{D}:=\mathscr{H}(\mathbf{R})$ which is an $\mathbf{E W}^{*}$-algebra and which contains the restrictions to $D$. of the position operator $t$ and the momentum operator $-\mathrm{i} d / d t$. A general result which supports the ahove conviction is contained in [9]. Roughly speaking and somewhat simplified, it says that if $\mathcal{A}$ is an $\mathrm{EW}^{*}$-algebra which is "realized" as an *-algebra of operators on a Hilbert space and which contains at least one unbounded operator, then the bounded part of $\mathcal{A}$ is necessarily a finite $W^{*}$-algebra.

In the present paper we go the second way by incorporating more general objects, than operators: continuous sesquilinear forms. To describe a typical object, suppose. $\mathscr{A}_{1}$ and $\mathscr{A}_{2}$ are $O^{*}$-algebras on domains $\mathscr{D}_{1}$ and $\mathscr{D}_{2}$, respectively, of a Hilbert space $\mathscr{H}$. If $a_{1} \in \mathcal{A}_{1}, a_{2} \in \mathcal{A}_{2}$, and $x \in B(\mathscr{H})$, then $\mathfrak{c}(\varphi, \psi):=\left\langle x a_{1} \varphi, a_{2} \psi\right\rangle, \varphi \in \mathscr{D}_{1}$ and $\psi \in \mathscr{D}_{2}$, defines a continuous sesquilinear form on $\mathscr{D}_{1}\left[\mathrm{t}_{\mathcal{A}_{1}}\right] \times \mathscr{D}_{2}\left[\mathrm{t}_{\mathcal{A}_{1}}\right]$. We shall denote this form by $c_{a_{a}+\circ x \circ a_{1}}$. The form $\mathfrak{c} \equiv \mathfrak{c}_{a_{2}+\circ x \circ a_{1}}$ is generated by an operator on $\mathcal{D}_{1}$ (in the sense that there is a linear operator $T$ defined on $\mathscr{D}_{1}$ such that $\mathfrak{c}(\varphi, \psi)=\langle T \varphi, \psi\rangle$ for all $: \varphi \in \mathscr{D}_{1}$ and $\left.\psi \in \mathscr{D}_{2}\right)$ if and only if $x a_{1} \mathscr{D}_{1} \subseteq \mathcal{D}\left(\left(a_{2}{ }^{+}\right)^{*}\right)$. The latter condition is, in general, not fulfilled and difficult to check. The basic object's investigated in this paper

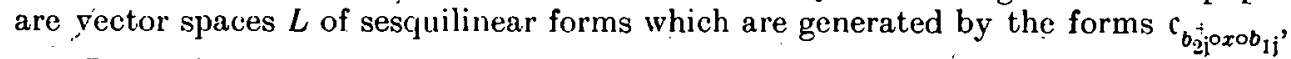
$x \in \mathscr{B}$ and $j \in \mathfrak{F}$. Here $\mathscr{B}$ is a (fixed) *-subalgebra of $\mathbf{B}(\mathscr{H})$ and $\left\{b_{1 \mathrm{j}} ; \mathrm{j} \in \mathfrak{Y}\right\}$ and $\left\{b_{2 j} ; \mathrm{i} \in \mathfrak{J}\right\}$ are indexed subsets of $\mathcal{A}_{1}$ and $\mathcal{A}_{2}$, respectively, which satisfy some additịonal assumptions. One crucial assumption requires that for all $j \in \mathfrak{J}$ and $k=1,2$ $b_{k \mathrm{i}} \mathscr{D}_{k}$ is dense in $\mathscr{H}$ and that $\overline{b_{k \mathrm{j}}}$ has a bounded inverse which belongs to $\mathscr{B}$.

The paper is organized as follows. In Section 1 we collect the basic definitions and some general facts needed in the sequel. In Section 2 we obtain two versions, of the von Neumann bicommutant theorem for spaces of sesquilinear forms. In Section 3 we show that the vector space of all $\mathrm{c}_{I \circ x \circ I}(\cdot, \cdot) \equiv\langle x \cdot, \cdot\rangle, x \in \mathscr{B}$, is dense in $\mathscr{L}\left[\tau_{1 \mathrm{n}}\right]$. This result is essentially used in Section 4 to prove a generalization of the Kaplansky density theorem to spaces of sesquilinear forms.

Vector spaces of continuous sesquilinear forms which are associated with unbounded operator algebras have becn already considered in several papers such as $[1,7,10,11,13]$. Condition (I) (in a slightly stronger form) first appeared in [1].

\section{Preliminaries}

Let $\mathscr{H}$ be a complex Hilbert space. The scalar product of $\mathscr{H}$ is always denoted by $\langle\cdot, \cdot\rangle$ and it is assumed to be linear in the first variable. Let $D$ be a dense linear subspace of $\mathscr{H}$ and let $\mathscr{L}^{+}(\mathscr{D}):=\left\{a \in\right.$ End $\mathscr{D} ; \mathscr{D} \subseteq \mathscr{D}\left(a^{*}\right)$ and $a^{*} \mathscr{D} \subseteq \mathscr{D}$. Then $\mathscr{L}^{+}(\mathscr{D})$ becomes an $*$-algebra if we take the composition of the operators as the multiplication and the involution $a \rightarrow a^{+}:=a^{*} \uparrow \mathscr{D}$. An $O^{*}$-algebra $\mathcal{A}$ on the domain $\mathcal{D}^{*}$ is an *-subalgebra of $\mathscr{L}^{*}(\mathscr{D})$ which contains the identity map $I$ of $\mathscr{D}$. Suppose that $\mathcal{A}$ is an 
$O^{*}$-algebra on $\mathscr{D}$. The graph topology $\mathrm{t}_{\mathcal{U}}$ is the locally convex topology on $\mathscr{D}$ which is defined by the family of seminorms $\varphi \rightarrow\|a \varphi\|, a \in \mathcal{A}$. We let $\mathscr{L}_{\mathscr{A}}^{+}(\mathscr{D})$ be the set of all $a \in \mathscr{L}^{+}(\dot{D})$ for which, $a$ and $a^{+}$map the locally convex space $\mathscr{D}\left[\mathrm{t}_{\mathscr{A}}\right]$ continuously into itself. Clearly, $\mathscr{L}_{\mathcal{A}}^{2}(\mathcal{D})$ is an $O^{*}$-algebra on $\mathcal{D}$. The $O^{*}$-algebra $\mathcal{A}$ is said to be closed on $\mathscr{D}$ if $\mathscr{D}=\cap\{\mathcal{D}(a) ; a \in \mathcal{A}\}$. Further, let $\mathcal{A}_{I}:=\{a \in \mathcal{A} ;\|\varphi\| \leqq\|a \varphi\|$ for $\varphi \in \mathscr{D}\}$.

Now we introduce some spaces of sesquilinear forms associated with unbounded operator algebras. In what follows suppose that $\mathcal{A}_{1}$ and $\mathcal{A}_{2}$ are $O^{*}$-algebras on domains $\mathscr{D}_{1}$ and $\mathscr{D}_{2}$, respectively, of the same Hilbert space $\mathscr{H}$. Let $\overline{D_{2}{ }^{\prime}}$ denote the complexconjugate vector space of the vector space $\mathscr{D}_{2}^{\prime}:=\mathscr{D}_{2}\left[\mathrm{t}_{\mathcal{A}_{\mathrm{x}}}\right]^{\prime}$. That is, $\overline{\mathcal{D}_{2}^{\prime}}$ equals $\mathscr{D}_{2}{ }^{\prime}$ as a set, the addition in $\mathscr{D}_{2}{ }^{\prime}$ is the same as in $\mathscr{D}_{2}{ }^{\prime}$, but the multiplication by 'scalars in $\mathscr{D}_{2}{ }^{\prime}$ 'is replaced in $\overline{D_{2}{ }^{\prime}}$ by the mapping $(\lambda, \varphi) \rightarrow \bar{\lambda}, q, \lambda \in \mathbf{C}$ and $\varphi \in \overline{D_{2}{ }^{\prime}}$. The mapping $\varphi \rightarrow\langle\cdot, \varphi\rangle$ is a linear injection of the Hilbert space $\mathscr{H}$ into the vector space $\overline{D_{2}{ }^{\prime}}$. Having this in mind, we use the notation $\langle\psi, \varphi\rangle$ also to denote the value of an arbitrary: linear functional $\varphi$ from $\mathscr{D}_{2}{ }^{\prime}$ at $\psi \in \mathscr{D}_{2}$ and we write $\langle\varphi, \psi\rangle$ for $\langle\overline{\psi, \varphi}\rangle$. Let $\mathscr{L}_{\mathcal{A}_{1}, \mathcal{A}_{2}}\left(\mathscr{D}_{1}, \mathscr{D}_{2}{ }^{\prime}\right)$ bé the vector space of all lineair mappings of $\mathscr{D}_{1}^{\prime}$ into $\overline{D_{2}{ }^{\prime}}$ for which the associated sesquilinear form $\mathfrak{c}_{x}$ defined by $\mathfrak{c}_{x}(\varphi, \psi):=\langle x \varphi, \psi\rangle, \varphi \in \mathscr{D}_{1}$ and $\psi \in \mathscr{D}_{2}$, is continuous on $\mathscr{D}_{1}\left[\mathrm{t}_{\mathcal{C}_{1}}\right] \times \mathscr{D}_{2}\left[\mathrm{t}_{\mathcal{C}_{3}}\right]$, that is, there are $a_{1} \in \mathcal{A}_{1}$ and $a_{2} \in \mathcal{A}_{2}$ such that

$$
\left|c_{x}(\varphi, \psi)\right| \equiv|\langle x \varphi, \psi\rangle| \leqq\left\|a_{1} \varphi\right\|\left\|a_{2} \psi\right\| \text { for all } \varphi \in \mathscr{D}_{1}, \psi \in \mathscr{D}_{2} .
$$

(By a-sesquilinear form on $\mathscr{D}_{1} \times \mathscr{D}_{2}$ we mean a complex-valued function on $\mathscr{D}_{1} \times \mathscr{D}_{2}$ which is linear in the first and conjugate-linear in the second variable.) The mapping $x \rightarrow \mathrm{c}_{x}$ is a linear bijection of $\mathscr{L}_{\mathcal{A}_{1}, \mathcal{A}_{1}}\left(\mathscr{D}_{1}, \mathscr{D}_{2}{ }^{\prime}\right)$ onto the rector space of all continuous sesquilinear forms on $\mathscr{D}_{1}\left[\mathrm{t}_{\mathcal{A}_{1}}\right] \times \mathscr{D}_{2}\left[\mathrm{t}_{\mathcal{A}_{2}}\right]$. (We prove the latter. It suffices to check that this map is surjective. For let $c$ be a continuous sesquilinear form on $\mathscr{D}_{1}\left[\mathrm{t}_{\mathcal{A}_{1}}\right] \times \mathscr{D}_{2}\left[\mathrm{t}_{\mathcal{A}_{2}}\right]$. Then, for each $\varphi \in D_{1}, \overline{c(\varphi, \cdot)}$ is in $D_{2}{ }^{\prime}$, so that $\overline{c(\varphi, \psi)}=\langle\psi, \tilde{\varphi}\rangle$ for some $\tilde{\varphi} \in \mathscr{D}_{2}{ }^{\prime}$. It is obvious that $\tilde{\varphi}$ is uniquely determined by $\varphi$. Putting $x \dot{\varphi}=\tilde{\varphi}$ for $\varphi \in \mathscr{D}_{1}, x$ is in $\mathscr{L}_{\mathcal{A}_{1}, \mathcal{A}_{1}}\left(\mathscr{D}_{1}, \mathscr{D}_{2}{ }^{\prime}\right)$ and $\mathrm{c}_{x}=$ c.)

We need somé more notation concerning the spaces $\mathscr{L}_{\mathcal{A}_{1}, \mathcal{A}_{1}}\left(\mathscr{D}_{1}, \mathscr{D}_{2}{ }^{\prime}\right)$. Let $\mathcal{A}$ be an $O^{*}$-algebra on $\mathscr{D}$. We write $\mathscr{L}_{\mathcal{A}}\left(\mathscr{D}, \mathscr{D}^{\prime}\right)$ for $\mathscr{L}_{\mathcal{A}, \mathcal{A}}\left(\mathscr{D}, \mathscr{D}^{\prime}\right)$ and $\mathscr{L}_{\mathcal{A}}(\mathscr{D}, \mathscr{H})$ for $\mathscr{L}_{\mathcal{A}, \mathrm{B}(\mathscr{H})}(\mathscr{D}, \mathscr{H})$. (This notation is not ambiguous, since if $D^{\prime}=\mathscr{H}$, then all operators in $\mathcal{A}$ are bounded, so that $\mathscr{L}_{\mathcal{A} \mathscr{A}}\left(\mathscr{D}, \mathscr{D}^{\prime}\right)=\mathscr{L}_{\mathcal{A}, \mathbf{B}(\mathscr{H})}(\mathscr{D}, \mathscr{H})$ in this case.) For $a_{1} \in \mathcal{A}_{1}$ and $a_{2} \in \mathcal{A}_{2}$, let

$$
\mathcal{U}_{a_{1}, a_{2}}:=\left\{x \in \mathscr{L}_{\mathcal{A}_{1}, \mathcal{A}_{3}}\left(\mathscr{D}_{1}, \mathscr{D}_{2}{ }^{\prime}\right) ;|\langle x \varphi ; \psi\rangle| \leqq\left\|a_{1} \varphi\right\| ! \mid a_{2} \psi \| \quad \text { for } \quad \varphi \in \mathscr{D}_{1}, \psi \in \mathscr{D}_{2}\right\} .
$$

We abbreviate $u_{a}:=\mathcal{U}_{a, a}, a \in \mathcal{A}$.

Next we define some locally convex topologies which are needed in the sequel.

The weak-operator topology on $\mathscr{L}_{\mathcal{A}_{1}, \mathcal{A}_{2}}\left(\mathscr{D}_{1}, \mathscr{D}_{2}{ }^{\prime}\right)$ is the locally convex topology which is generated by the family of seminorms

$$
x \rightarrow|\langle x \varphi, \psi\rangle|, \quad \varphi \in \dot{D}_{1} \text { and } \psi \in \mathscr{D}_{2} \text {. }
$$

- For an $O^{*}$-algebra $\mathcal{A}$ on $\mathcal{D}$, let $l_{2}(\mathcal{A})$ denote the set of all sequences $\left(\varphi_{n} ; n \in \mathrm{N}\right)$ from $\mathscr{D}$ for which $\left(\left\|a \varphi_{n}\right\| ; n \in \mathbf{N}\right)$ is in $l_{2}(\mathbf{N})$ for all $a \in \mathcal{A}$. The ultraweak topology on $\mathscr{L}_{\boldsymbol{d}_{1}, \mathcal{A}_{1}}\left(\mathscr{D}_{1}\right.$, $D_{2}^{\prime}$ ) is the locally convex topology which is defined by the seminorms

$$
x \rightarrow\left|\sum_{n=1}^{\infty}\left\langle x \varphi_{n}, \psi_{n}\right\rangle\right|, \quad\left(\varphi_{n}\right) \in l_{2}\left(\mathcal{A}_{1}\right) \text { and }\left(\psi_{n}\right) \in l_{2}\left(\mathscr{A}_{2}\right) \text {. - }
$$

(Since, by definition, each $x \in \mathscr{L}_{\mathcal{L}}, \mathcal{A}_{1}\left(\mathscr{D}_{1}, \mathcal{D}_{2}{ }^{\prime}\right)$. satisfies (1.1) for some $a_{1} \in \mathcal{A}_{1}$ and $a_{2} \in \mathcal{A}_{2}$, it follows from the Cauchy-Schwarz inequality that the infinite sum in (1.2) converges.) If ambiguities can occur, we speak about the weak-operator topology or the ultraweak topology with respect to $\mathscr{D}_{1} \times \mathscr{D}_{8}$. If $\mathscr{S}_{1} \cong \mathscr{H}$ and $\mathscr{S}_{2} \subseteq \mathscr{H}$, then the 
weak-operator topology on $\mathrm{B}(\mathscr{H})$ with respect to $\mathscr{G}_{1} \times \mathscr{G}_{2}$ is defined by the seminorms $x \rightarrow|\langle\dot{x} \varphi, \psi\rangle|, \varphi \in \mathscr{G}_{1}$ and $\psi \in \mathscr{S}_{2}$. Let $\mathcal{A}$ be an $O^{*}$-algebra on $D$. The strongoperator topology and the ultrastrong topology on $\mathscr{L}_{\mathcal{M}}(\mathcal{D}, \mathscr{H})$ are defined by the families of seminorms

. $\quad \therefore \quad x \rightarrow\|x \varphi\|, \varphi \in \mathcal{D}$, and $x \rightarrow\left(\sum_{n=1}^{\infty}\left\|x \varphi_{n}\right\|^{2}\right)^{1 / 2},\left(\varphi_{n}\right) \in l_{2}(\mathcal{A})$,

respectively.

Suppose $\mathscr{L}$ is a linear subspace of $\mathscr{L}_{\mathcal{A}_{1}, \mathcal{A}_{2}}\left(\mathscr{D}_{1}, \mathscr{D}_{2}{ }^{\prime}\right)$. For $a_{1} \in \mathcal{A}_{1}$ and $a_{2} \in \dot{\mathcal{A}_{2}}$, let $\mathscr{L}_{a_{1}, a_{3}}$ be the'set of all $x \in \mathscr{L}$ for which there exists a positive number $\lambda$ such that

$$
|\langle x \varphi, \psi\rangle| \leqq \lambda\left\|a_{1} \varphi\right\|\left\|a_{2} \psi\right\| \text { for all } \varphi \in \mathscr{D}_{1} \text { and } \psi \in \mathscr{D}_{2} \text {. }
$$

If $x \in \mathscr{L}_{a_{1}, a_{1}}$, let $l_{a_{1}, a_{8}}(x)$ be the infimum of all $\lambda>0$ for which (1.3) is satisfied. Obviously, $\mathscr{L}_{a_{1}, a}$ is a linear subspace of $\mathscr{L}$ and $l_{a_{1}, a}(\cdot)$ is a norm on $\mathscr{L}_{a_{1}, a_{k}}$. Because of the definition of $\mathscr{L}_{\mathcal{A}_{1}, \mathcal{A}_{1}}\left(\mathscr{D}_{1}, \mathscr{D}_{2}{ }^{\prime}\right)$, we have $\mathscr{L} \doteq \bigcup\left\{\mathscr{L}_{a_{1}, a_{1}} ; a_{1} \in \mathcal{A}_{1}, a_{2} \in \mathcal{A}_{2}\right\}$. Let $\tau_{\text {in }}$ denote the inductive topology on $\mathscr{L}$ with respect to the embedding maps $i_{a_{1}, a_{2}}:\left(\mathscr{L}_{a_{1}, a_{2}}, l_{a_{1}, a_{3}}\right)$ $\rightarrow \mathscr{L}, a_{1} \in \mathcal{A}_{1}$ and $a_{2} \in \mathcal{A}_{2}$. That is, $\tau_{\text {in }}$ is the finest locally convex topology on $\mathscr{L}$ for which all mappings $i_{a_{1}, a_{2}}, a_{1} \in \mathcal{A}_{1}$ and $a_{2} \in \mathcal{A}_{2}$, are continuous. The topologies $\varrho$ and $\lambda$ as defined in [2] appear as special cases of this topology $\tau_{\mathrm{in}}$. This and some other aspects of the topology $\tau_{\text {in }}$ will be discussed elsewhere.

As in [1] and in [10], we define a partial multiplication in $\mathscr{L}_{\mathcal{A}_{1}, \mathcal{A}_{8}}\left(\mathscr{D}_{1}, \mathscr{D}_{2}{ }^{\prime}\right)$. Suppose that $y \in \mathscr{L}_{\mathcal{A}_{1}, \mathcal{A}_{1}}\left(\mathscr{D}_{1}, \mathscr{D}_{2}{ }^{\prime}\right), \dot{a}_{1} \in \mathscr{L}_{\mathcal{A}_{1}}^{+}\left(\mathscr{D}_{1}\right)$ and $a_{2} \in \mathscr{L}\left(\dot{\mathcal{U}}_{3} \mathscr{D}_{2}\right)$. Obviously, $c(\varphi, \psi):=\left\langle x a_{1} \varphi\right.$, $\left.a_{2} \psi\right\rangle, \varphi \in D_{1}$ and $\psi \in \mathscr{D}_{2}$, defines a continuous sesquilinear form on $\mathscr{D}_{1}\left[t_{\mathcal{A}_{1}}\right] \times \mathscr{D}_{2}\left[t_{\mathcal{A}_{3}}\right]$. Hence there is an $x \in \mathscr{L}_{\mathcal{U}_{1}, \mathcal{A}_{2}}\left(\mathcal{D}_{1}, \mathscr{D}_{2}{ }^{\prime}\right)$ such that $c=c_{x}$. Define $a_{2}{ }^{+} \circ y \circ a_{1}:=x$. That is, by definition, we have

$$
\left\langle\left(a_{2}{ }^{+} \circ y \circ a_{1}\right) \varphi, \psi\right\rangle=\left\langle y a_{1} \varphi, a_{2} \psi\right\rangle \text {. for } \quad \varphi \in \mathscr{D}_{1} \text { and } \psi \in \mathscr{D}_{2} \text {. }
$$

Let $a_{1}$ and $a_{2}$ be as above and let $y \in \mathbf{B}(\mathscr{H})$. Since, in particular, $y \uparrow \mathscr{D} \in \mathscr{L}_{\mathcal{A}_{1}, \mathcal{A}_{1}}\left(\mathscr{D}_{1}, \mathscr{D}_{2}{ }^{\prime}\right)$, $a_{2}{ }^{+} \circ(y \uparrow D) \circ a_{1}$ is well-defined by the preceding. For notational'simplicity we write $a_{2}{ }^{+} \circ y \circ a_{1}$ instead of $a_{2}{ }^{+} \circ(y \uparrow \mathcal{D}) \circ a_{1}$. If $\mathscr{B} \subseteq \mathbf{B}(\mathscr{H})$, then $a_{2}{ }^{+} \circ \mathscr{B} \circ a_{1}$ denotes the set of all $a_{2}{ }^{+} \circ y \circ a_{1}$, where $y \in \mathscr{B}$.

The following simple lemma will be needed several times. In the special case $a_{1}=a_{2}$ it is stated as Proposition 5.1 in [10]. The proof in the general case can be given by a slight modification of the proof of Proposition 5.1 in [10], so it will be omitted.

I.emma 1: Suppose $x \in \mathscr{L}_{\mathcal{A}_{1}, \mathcal{A}_{2}}\left(\mathscr{D}_{1}, \mathcal{D}_{2}{ }^{\prime}\right), a_{1} \in\left(\mathcal{A}_{1}\right)_{I}$ and $a_{2} \in\left(\mathcal{A}_{2}\right)_{I}$. Assume that there is a constant $\lambda$ such that (1.3) is satisfied. Then there exists an operator $y \in \mathbf{B}(\mathscr{K})$ such that $x=a_{2}^{+} \circ y \circ a_{1}$.

\section{The von Neumann bicommutant theorem for spaces of sesquilinear forms}

Let $\mathcal{A}$ be an $O^{*}$-algebra on a domain $\mathcal{D}$. For subsets $\mathcal{M} \subseteq \mathscr{L}_{\mathcal{A}}\left(\mathcal{D}, \mathcal{D}^{\prime}\right)$ and $\mathcal{N} \subseteq \mathscr{L}_{\mathcal{A}}(\mathcal{D})$, we define "commutants" $\mathscr{M}^{3}$ and $\mathscr{N}^{\mathrm{c}}$ by

and

$$
\mathscr{M}^{\mathrm{J}}:=\left\{a \in \mathscr{L}_{\mathcal{L}}^{+}(\mathscr{D}) ; x \circ a=a \circ \dot{x} \text { for all } x \in \mathscr{M}\right\}
$$

$$
\mathscr{N}^{\mathrm{c}}:=\left\{x \in \mathscr{P}_{\mathfrak{A}}\left(\mathcal{D}, \mathscr{D}^{\prime}\right) ; x \circ a=a \circ x \text { for all } a \in \mathscr{N}\right\} \text {. }
$$

Further, let $\mathcal{M}_{\mathrm{b}}$ denote the set of all bounded operators in $\mathscr{M}^{\mathrm{s}}$. (It should be noted that the notation concerning commutants of unbounded operator algebras is not yet 
standard. For instance, our notation differs from the one used in $[1,5,14])$. In the above notation, we have

Theorem $1:$ Suppose $\mathcal{A}$ is a closed $O^{*}$-algebra on $\mathcal{D}$. Suppose that there exists a subset $\left\{b_{\mathrm{j}} ; \mathrm{j} \in \mathfrak{\Im}\right\}$ of operators from $\mathcal{A}_{I}$ such that $b_{\mathrm{i}} \mathcal{D}$ is dense in $\mathscr{H}$ for each $\mathrm{j} \in \mathfrak{\Im}$ and such that $\|\cdot\|_{b_{\mathfrak{j}}}, \mathrm{j} \in \mathfrak{S}$, is a directed family of seminorms which generates the graph topology $\mathrm{t}_{\mathcal{A}}$ on $\mathcal{D}$. Suppose $\mathscr{B}$ is an *-subalgebra of $\mathbf{B}(\mathscr{H})$ which contains all operators $\left(\overline{b_{\mathrm{j}}}\right)^{-1}, \mathrm{j} \in \mathfrak{\Im}$. Let $\mathscr{L}$ be the linear hull of $b_{\mathrm{i}}^{+} \circ \mathscr{B} \circ b_{\mathrm{i}}$, $\mathrm{i} \in \mathfrak{J}$, in $\mathscr{L}_{\mathcal{A}}\left(\mathscr{D}, \mathscr{D}^{\prime}\right)$.

Then $\left(\mathscr{S}_{\mathrm{b}}^{\mathrm{D}}\right)^{\mathrm{c}}$ coincides with the ultraweak closure of $\mathscr{L}$ within $\mathscr{L}_{\mathrm{d}}\left(\mathscr{D}, \mathscr{D}^{\prime}\right)$. Moreover, $\left(\mathscr{L}_{\mathrm{b}}^{\mathrm{D}}\right)^{\mathrm{c}} \doteq\left(\mathscr{L}^{\mathrm{J}}\right)^{\mathrm{c}}=\bigcup_{\mathrm{j} \in \mathfrak{Y}} b_{\mathrm{j}}^{+} \circ \mathscr{B}^{\prime \prime} \circ b_{\mathrm{j}}$.

We first prove the following simple

Lem ma 2: Let $\mathcal{A}$ be an $O^{*}$-algebra on $\mathcal{D}$ and let $a$ and $b$ be operators from $\mathcal{A}$ such that

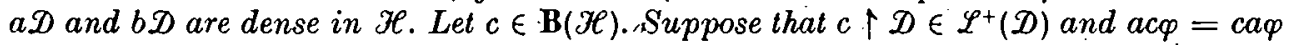
and $b c^{*} \varphi=c * b \varphi$ for $\varphi \in \mathcal{D}$. Let $z:=b^{+} \circ x \circ a$, where $x \in \mathbf{B}(\mathscr{H})$.

Then $c \circ z=z \circ c$ if and only if $c x=x c$.

Proof: For $\varphi, \psi \in \mathcal{D}$, we have by definition,

and

$$
\left.\langle c \circ z \varphi, \psi\rangle=\left\langle z \varphi, c^{*} \psi\right\rangle=\left\langle x a \varphi, b c^{*} \psi\right\rangle\right\rangle^{\prime}=\left\langle a \varphi, x^{*} c^{*} b \psi\right\rangle
$$

$$
\langle z \circ c \varphi, \psi\rangle=\langle\dot{x} a c \varphi, b \psi\rangle=\left\langle a \varphi, c^{*} x^{*} b \psi\right\rangle \text {. }
$$

Here we used èssentially the commutativity assumptions concerning $a, c$ and $b, c^{*}$. Since $a \mathscr{D}$ and $b D$ are assumed to be dense in $\mathscr{H}$, we conclude from (2.1) and (2.2) that $-c \circ z=z \circ c$ if and only if $x^{*} c^{*}=c^{*} x^{*}$, that is, if $c x=x c$

Proof of Theorem 1: First we check that $\mathscr{B}^{\prime} \uparrow \mathscr{D} \subseteq \mathscr{L}_{\mathfrak{A}}^{+}(\mathscr{D})$. Fix $x \in \mathscr{B}^{\prime}$. Since $\left(\overline{b_{\mathrm{j}}}\right)^{-1} \in \mathscr{B}, x\left(\overline{b_{\mathrm{i}}}\right)^{-1}=\left(\overline{b_{\mathrm{i}}}\right)^{-1} x$ and hence $x \overline{b_{\mathrm{i}}} \subseteq \bar{b}_{\mathrm{j}}^{\prime} x$ for $\mathrm{j} \in \mathfrak{\Im}$. In particular, $x \mathcal{D}$ $\subseteq x \mathscr{D}\left(\overline{b_{\mathrm{j}}}\right) \subseteq \mathcal{D}\left(\overline{b_{\mathrm{j}}}\right)$ for $\mathrm{i} \in \mathfrak{\Im}$. Since $\mathcal{A}$ is closed on $\mathcal{D}$ and the family of seminorms $\|\cdot\|_{b_{\mathfrak{j}}}$, i $\epsilon \mathfrak{J}$, is directed and generates $\mathrm{t}_{\mathcal{A}}$; we have $\mathscr{D}=\cap\left\{\mathscr{D}\left(b_{\mathrm{i}}\right) ; \mathrm{i} \in \mathfrak{I}\right\}$. Therefore, $x \mathscr{D} \subseteq \mathscr{D}$. Because $\mathscr{B}$ is an $*$-algebra, $x^{*} \mathscr{D} \subseteq \mathcal{D}$ and so $x^{\prime} \uparrow \mathcal{D} \in \mathscr{L}+(\mathscr{D})$. Since $x$ and $x^{*}$ commute with $b_{\mathfrak{i}}, \mathrm{i} \in \mathfrak{I}$, on $\mathcal{D}$ and since $\dot{\mathrm{t}}_{\mathscr{A}}$ is generated by $\|\cdot\|_{b_{\mathfrak{j}}}, \mathrm{j} \in \mathfrak{J}$, it follows that $x \in \mathscr{L}_{\mathfrak{A}}^{+}(\mathscr{D})$.

Next we prove that $\mathscr{B}^{\prime} \uparrow \mathcal{D}=\mathscr{P}_{\mathrm{b}}^{\circ}$. Let $\mathrm{i} \in \mathfrak{\Im}$. It is straightforward to verify that $b_{\mathrm{i}}{ }^{+} \circ\left(\overline{b_{\mathrm{j}}}\right)^{-1} \circ b_{\mathrm{j}}=b_{\mathrm{i}}^{+}$and $\left.b_{\mathrm{i}}{ }^{+} \circ\left(\overline{\left(b_{\mathrm{i}}\right.}\right)^{-1}\right)^{*} \circ b_{\mathrm{i}}=b_{\mathrm{i}}$. Therefore, because $\mathscr{B}$ is an *-algebra and $\left(\bar{b}_{j}\right)^{-1} \circ \mathscr{B}_{\text {; }}$ the operators $b_{\mathrm{j}}$ and $b_{\mathrm{j}}^{+}$are in $\mathscr{L} \cap \mathscr{L}_{\mathcal{A}}^{+}(\mathcal{D})$. Suppose $x \in \dot{\mathscr{L}}_{0}$. Then $x$ commutes with $b_{\mathrm{i}}$ and $b_{\mathrm{i}}{ }^{+}$on $\mathscr{D}$. Since' $x \in \mathscr{P}_{\mathcal{A}}^{+}(\mathcal{D})$, this implies that $x^{+}=(x)^{*} \Gamma \mathcal{D}$ commutes with $b_{\mathrm{i}}$ as well. Therefore, applying Lemma 2 in case $a=b=b_{\mathrm{i}}$, we get $x \in \mathscr{B}^{\prime}$. This shows that $\mathscr{L}_{\mathrm{b}} \subseteq \mathscr{B}^{\prime} \uparrow \mathscr{D}$. Conversely, suppose $x \in \mathscr{B}^{\prime}$. As shown above, $x \uparrow \mathscr{D} \in \mathscr{L}_{\mathcal{A}}(\mathscr{D})$ and $x \uparrow \mathscr{D}^{\circ}$ commutes with $b_{\mathrm{j}}$ and $b_{\mathrm{i}}{ }^{+}$. on $\mathcal{D}$ for each $\mathrm{i} \in \mathfrak{\Im}$. "The same is true for $x^{*} \uparrow \mathscr{D}$. Thus, again by Lemma $2, x, \mathfrak{D} \in \mathscr{L}_{\mathrm{b}}^{\circ}$. Hence $\mathscr{B}^{\prime} \uparrow \mathscr{D}=\mathscr{L}_{\mathrm{b}}$.

Suppose that $z \in\left(\mathscr{L}_{\mathrm{b}}^{\mathrm{P}}\right)^{\mathrm{c}}$. From Iemma 1.1 and the assumptions, there are an index $\mathrm{j} \in \mathfrak{J}$ and a bounded operator $x$ on $\mathscr{X}$ such that $z=b_{\mathrm{j}}{ }^{+} \circ x \circ b_{\mathrm{j}}$. Applying Lemma 2 once more, we conclude that $x$ commutes with the closures of the operators from $\mathscr{L}_{\mathrm{b}}=\mathscr{B}^{\prime} \uparrow \mathscr{D}$. Hence $x \in \mathscr{B}^{\prime \prime}$. Since $\left(\bar{b}_{\mathrm{i}}\right)^{-1} \in \mathscr{B}$ for $\mathrm{j} \in \mathfrak{J}_{,} \mathscr{B}$ is a non-degenerate *-subalgebra of $\mathbf{B}(\mathscr{H})$, so that the von Neumann density theorem applies (see e.g. $\left[17 ;\right.$ p. 74]). There exists a net $\left\{x_{1}\right\}$ of operators from $\mathscr{B}$ which converges to $x$ in the ultraweak topology on $\mathscr{H}$. This implies that the net $\left\{b_{\mathrm{i}}^{+} \circ x_{\mathfrak{l}} \circ b_{\mathrm{i}}\right\}$ converges to $b_{\mathrm{i}}^{+}$ $\circ x \circ b_{\mathrm{j}}=z$ in the ultraweak topology on $\mathscr{D}$. Since $b_{\mathrm{j}}{ }^{+} \circ x_{1} \circ b_{\mathrm{j}} \in \mathscr{L}$ for all $\mathfrak{l}$, $z$ belongs to the ultraweak closure $\overline{\mathscr{L}}^{\text {uw }}$ of $\mathscr{L}$ within $\mathscr{L}_{\mathcal{M}}\left(\mathscr{D}, \mathcal{D}^{\prime}\right)$. Thus we have shown that 


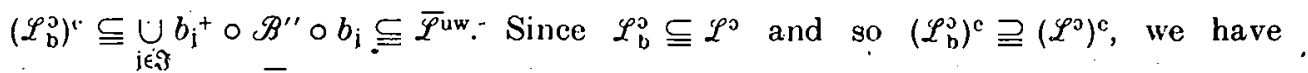
$\left(\mathscr{L}^{\mathrm{O}}\right)^{\mathrm{c}} \subseteq\left(\mathscr{L}_{\mathrm{b}}^{\mathrm{j}}\right)^{\mathrm{c}} \subseteq \overline{\mathscr{L}}^{\text {uw }}$. Since obviously $\mathscr{L} \subseteq\left(\mathscr{L}^{\mathrm{j}}\right)^{\mathrm{c}}$ and $\left(\mathscr{L}^{\mathrm{j}}\right)^{\mathrm{c}}$ is ultraweakly closed in $\mathscr{L}_{\mathcal{A}}\left(\mathscr{D}, \mathscr{D}^{\prime}\right)$, the preceding gires $\left(\mathscr{L}^{\mathrm{J}}\right)^{\mathrm{c}}=\left(\mathscr{L}_{\mathrm{b}}^{\mathrm{a}}\right)^{\mathrm{c}}=\bigcup_{\mathrm{i} \in \mathfrak{S}} b_{\mathrm{i}}^{+} \circ \mathscr{B}^{\prime \prime} \circ b_{\mathrm{i}}=\overline{\mathscr{L}}^{\mathrm{uw}}$.

The next theorem contains a similar result for the ultrastrong-operator topology. For a subset $\mathscr{N}$ of $\mathscr{L}_{\mathcal{A}}^{+}(\mathscr{D})$, let

$$
\mathscr{N}_{\mathrm{w}}^{\mathrm{c}}:=\left\{x \in \mathscr{P}_{\mathfrak{A}}(\mathcal{D}, \mathscr{H}):\langle x a \varphi, \psi\rangle=\left\langle x \varphi ; a^{+} \psi\right\rangle \text { for all } \varphi, \dot{\psi} \in \mathcal{D}, a \in \mathscr{N}\right\}
$$

Theorem 3: Let $\mathcal{A},\left\{b_{\mathrm{j}} ; \mathrm{i} \in \mathfrak{S}\right\}$ and $\mathscr{B}$ sutisfy the assumptions of Theorem 1 . Assume in addition that $I \in \mathscr{B}$. Let $\mathscr{L}$ be the linear span of $x b_{\mathrm{i}}$, where $x \in \mathscr{B}$ and $\mathrm{j} \in \mathfrak{\Im}$.

Then $\left(\mathscr{L}_{\mathrm{b}}^{\mathrm{D}}\right)_{\mathrm{w}}^{\mathrm{c}}$ is the ultrastrong closure of $\mathscr{L}_{\text {in }} \mathscr{L}_{\mathfrak{A}}(\mathscr{D}, \mathscr{H})$ and $\left(\mathscr{L}_{\mathrm{b}}^{\mathrm{D}}\right)_{\mathrm{w}}^{\mathrm{c}}=\left(\mathscr{L}^{\mathrm{D}}\right)_{\mathrm{w}}^{\mathrm{c}}=\bigcup_{\mathrm{i} \in \mathfrak{Y}} \mathscr{B}^{\prime \prime} b_{\mathrm{i}}$.

Proof: As in the proof of Theorem 1, we have $\mathscr{B}^{\prime} \uparrow \mathscr{D} \subseteq \mathscr{L}_{\mathcal{A}}^{+}(\mathscr{D})$ and $x b_{\mathrm{i}} \varphi=b_{\mathrm{j}} x \varphi$, $\varphi \in \mathscr{D}$, for $x \in \mathscr{B}^{\prime}$ and $\mathrm{j} \in \mathfrak{\Im}$. Since $I \in \mathscr{B}, b_{\mathrm{i}} \in \mathscr{L}$ for each $\mathrm{j} \in \mathfrak{\Im}$. Therefore, applying Lemma 2 in case $a=b_{1}, b=I$, we get $\mathscr{B}^{\prime} \uparrow \mathcal{D}=\mathscr{L}_{\mathrm{n}}$ similarly as in the proof of Theorem 1.

Suppose $z \in\left(\mathscr{L}_{\mathrm{b}}^{\mathrm{o}}\right)_{\mathrm{w}}^{\mathrm{c}}$. Since $z \in \mathscr{L}_{\mathcal{A}}(\mathscr{D}, \mathscr{H})$, there are $\mathrm{j} \in \mathfrak{J}$ and $x \in \mathbf{B}(\mathscr{H})$ such that $\dot{z}=x b_{\text {j. }}$. Employing again Lemma 2 , we get $x \in \mathscr{B}^{\prime \prime}$. By the von Neumann density theorem, there is a net $\left\{x_{1}\right\}$ from $\mathscr{B}$ converging to $x$ in the ultrastrong topology on $\mathscr{H}$. Then the net $\left\{x_{1} b_{\mathrm{j}}\right\}$ from $\mathscr{L}$ converges to $x b_{\mathrm{j}}=z$ in the ultrastrong topology on $\mathscr{D}$.

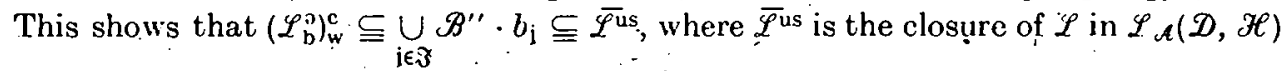
with respect to the ultrastrong topology. Since $\mathscr{L} \subseteq\left(\mathscr{L}^{0}\right)_{\mathrm{w}}^{\mathrm{c}} \subseteq\left(\mathscr{L}_{\mathrm{b}}^{J}\right)_{\mathrm{w}}^{\mathrm{c}}$ and since $\left(\mathscr{L}^{0}\right)_{\mathrm{w}}^{\mathrm{c}}$ is obviously ultrastrongly closed in $\mathscr{L}_{\mathcal{A}}(\mathcal{D}, \mathscr{H})$, the assertion follows

\section{Density of the bounded part}

Let $\mathcal{A}_{1}$ and $\mathcal{A}_{2}$ be $O^{*}$-algebras on domains $\mathcal{D}_{1}$ and $\mathscr{D}_{2}$, respectively, of the same Hilbert space $\mathscr{H}$ and let $\mathscr{B}$ be an $*$-subalgebra of $\mathbf{B}(\mathscr{H})$. Let $\mathfrak{J}$ be an index set. In order to formulate. Theorem 1 below and the results in Section 4, we need the following condition:

For $k \in\{1,2\}$ there exist a set $\left\{a_{k \mathrm{i}} ; \mathrm{i} \in \mathfrak{Y}\right\}$ of symmetric operators from $\mathscr{A}_{k}$ and $a$ set $\left\{\alpha_{k \mathrm{i}} ; \mathrm{i} \in \Im\right\}$ of complex numbers such that $b_{k \mathrm{i}}:=a_{k \mathrm{j}}+\alpha_{k \mathrm{j}} I$ belongs to $\left(\mathcal{A}_{k}\right)_{I}, b_{k \mathrm{j}} \mathscr{D}$ is dense in $\mathscr{H}$ and $B_{k \mathrm{j}}:=\left(\overline{b_{k \mathrm{j}}}\right)^{-1} \in \mathscr{B}$ for each $\mathrm{j} \in \mathfrak{J}$.

Note that (1) implies that the operators $\overline{a_{k j}}, \mathrm{j} \in \mathfrak{Y}$ and $k \in\{1,2\}$, are maximal symmetric, i.e., at least one of the deficiency indices of $\overline{a_{k j}}$ vanishes.

'Theorem 1: Let $\mathcal{A}_{1}, \mathcal{A}_{2}$ and $\mathscr{B}$ as above. Assume that (I) is fulfilled. Let $\mathscr{L}$ denote the linear span of $b_{2 \mathrm{j}}^{+} \circ \mathscr{B} \circ b_{1 \mathrm{i}}, \mathrm{j} \in \mathcal{J}$, in $\mathscr{L}_{\mathcal{A}_{1} \mathcal{A}_{\mathrm{z}}}\left(\mathscr{D}_{1}, \mathscr{D}_{2}{ }^{\prime}\right)$. Then $\mathscr{B} \uparrow \mathscr{D}$ is dense in $\mathscr{L}\left[\tau_{\text {in }}\right]$.

The proof of Theorem 1 is based on two auxiliary lemmas.

Lemma-2: Let a be a symmetric operator and let a be a complex number such that $\bar{a}+\alpha I$ has a bounded inverse on the underlying Hilbert space $\mathscr{H}$. Then, for each $\varepsilon>0$ and $\varphi \in \mathscr{H}$.

$$
\left\|(\bar{a}+\alpha I)^{-2} \varphi\right\|^{2} \leqq \varepsilon^{2}\|\varphi\|^{2}+\varepsilon^{-1}\left\|(\bar{a}+\alpha I)^{-3} \varphi\right\|^{2} .
$$


0

Proof: Upon extending $a$ to a self-adjoint operator in a possibly larger Hilbert space, we can assume without loss of generality that $a$ is self-adjoint. Fix $\varepsilon>0$ and let $e$ be the spectral projection of $a$ associated with the set $\left\{\hat{\lambda} \in \mathbf{R}:|\dot{\lambda}+\alpha|^{2} \geqq \varepsilon^{-1}\right\}$. By the spectral theorem,

and

$$
\varepsilon^{2}\|\varphi\|^{2} \geqq \varepsilon^{2}\|e \varphi\|^{2} \geqq\left\|(a+\alpha I)^{-2} e \varphi\right\|^{2}
$$

$$
\begin{aligned}
\varepsilon^{-1}\left\|(a+\alpha I)^{-3} \varphi\right\|^{2} & \geqq \varepsilon^{-1}\left\|(a \hat{+} \alpha I)^{-3}(I-e) \varphi\right\|^{2} \\
& \geqq\left\|(a+\alpha I)^{-2}(I-e) \varphi\right\|^{2}
\end{aligned}
$$

for $\varphi \in \mathscr{H}$ which implies (3.1)

The next lemma is a generalization of Lemma 6.1 in [1].

Lemma 3: Let $c_{1}$ and $c_{2}$ be positive operators from an *-subalgebra $\mathscr{B}$ of $\mathbf{B}(\mathscr{H})$. Suppose $\alpha_{1}, \alpha_{2} \in \mathbf{R}, 0<\alpha_{1} \leqq 1,0<\alpha_{2} \leqq 1$. Let $z$ be an operator from $\mathscr{B}$ satisfying

$$
|\langle z \varphi, \psi\rangle|^{2} \leqq\left\langle\left(c_{1}+\alpha_{1} I\right) \varphi, \varphi\right\rangle\left\langle\left(c_{2}+\alpha_{2} I\right) \psi, \psi\right\rangle \text { for } \varphi, \psi \in \mathscr{H}:
$$

Then there are operators $\ddot{z}_{1}, z_{2} \in \mathscr{B}$ such that $z=z_{1}+z_{2}$,

and

$$
\left|\left\langle z_{1} \varphi, \psi\right\rangle\right|^{2} \leqq\left\langle c_{1} \varphi, \varphi\right\rangle\left\langle\grave{c}_{2} \psi, \psi\right\rangle \text {. }
$$

$$
\left|\left\langle z_{2} \varphi, \psi\right\rangle\right| \leqq 2\left(\left(\alpha_{1} \alpha_{2}\right)^{1 / 2}+\left(\alpha_{1}\left\|c_{2}\right\|\right)^{1 / 2}+\left(\alpha_{2}\left\|c_{1}\right\|\right)^{1 / 2}\right)\|\varphi\|\|\psi\|
$$

for $\varphi, \psi \in \mathscr{H}$. Moreover, there is an operator $y_{1} \in \mathscr{B}$ such that $z_{1}=c_{2} y_{1} c_{1}$.

Proof:The proof is nothing but an adaptation of the proof of Lemma 6.1. in [1] to the present situation. Let $\lambda:=1 / \max \left(1,\left\|c_{1}\right\|,\left\|c_{2}\right\|\right)$. Upon replacing $z, c_{1}, c_{2}, \alpha_{1} ; \alpha_{2}$ by $\hat{i z}, \lambda c_{1}, \lambda c_{2}, \lambda \alpha_{1}, \lambda \alpha_{2}$, respectively, we can assume that $\left\|c_{1}\right\| \leqq 1$ and $\left\|c_{2}\right\| \leqq 1$. Fix $\alpha \in \mathbf{R}, 0<\alpha \leqq 1$. Let $f$ denote the function on $[0,1]$ which is defined by $f(t)$ $=(t(t+\alpha))^{-1 / 2}$ if $t \in[\varepsilon, 1]$ and $f(t)=(\varepsilon(\varepsilon+\alpha))^{-1 / 2}$ if $t \in[0, \varepsilon]$, where $\varepsilon$ is a positive number satisfying $4 \varepsilon \leqq \alpha^{1 / 2}$ and $\varepsilon \leqq \alpha$. We approximate the real continuous function $f-\varepsilon$ on $[0,1]$ by a real polynomial $p$ such that $|p(t)-f((t)-\varepsilon)| \leqq \varepsilon$ for $t \in[0,1]$. Put $q(t):=i p(t)$. It is easy to check that for $t \in[0,1]$

and

$$
-0 \leqq q(t) \leqq t^{1 / 2}(t+\alpha)^{-1 / 2}
$$

$$
0 \leqq(t+\alpha)^{1 / 2}(1-q(t)) \leqq 2 \alpha^{1 / 2}
$$

Suppose $k \in\{1,2\}$. Let $q_{k}$ be the polynomial $q$ defined above in case $\alpha \stackrel{i=}{=} \alpha_{k}$ and let $\underline{b}_{k}:=q_{k}\left(c_{k}\right)$. Define $z_{1}:=b_{2} z b_{1}$ and $z_{2}:=z-z_{1}$. Since $q_{1}$ and $q_{2}$ are polynomials with vanishing constant coefficients, $b_{1}=q_{1}\left(c_{1}\right) \in \mathscr{B}, b_{2}=q_{2}\left(c_{2}\right) \in \mathscr{B}$ and $z_{1}=c_{2} y_{1} c_{1}$ for some $y_{1} \in \mathscr{B}$. In particular; $z_{1} \in \mathscr{B}$ and $z_{2} \in \mathscr{B}$. If $\phi, \psi \in \mathscr{H}$, applying (3.2) and (3.5),

$$
\begin{aligned}
\left|\left\langle z_{1} \varphi, \psi\right\rangle\right|^{2}=\left|\left\langle z b_{1} \varphi, b_{2} \psi\right\rangle\right|^{2} & \leqq\left\langle\left(c_{1}+\alpha_{1} I\right) b_{1} \varphi, b_{1} \varphi\right\rangle\left\langle\left(c_{2}+\alpha_{2} I\right) b_{2} \psi, b_{2} \psi\right\rangle \\
& \leqq\left\langle c_{1} \varphi, \varphi\right\rangle\left\langle c_{2} \psi, \psi\right\rangle .
\end{aligned}
$$

From (3.2), (3.5) and (3.6)

$$
\begin{aligned}
\left|\left\langle z_{2} \varphi, \psi\right\rangle\right| \leqq & \left|\left\langle z b_{1} \varphi,\left(I-b_{2}\right) \psi\right\rangle\right|+\left|\left\langle z\left(I-b_{1}\right) \varphi, \psi\right\rangle\right| \\
\leqq & \left\langle\left(c_{1}+\alpha_{1} I\right) b_{1} \varphi, b_{1} \varphi\right\rangle^{1 / 2}\left\langle\left(c_{2}+\alpha_{2} I\right)\left(I-b_{2}\right) \psi,\left(I-b_{2}\right) \psi\right\rangle^{1 / 2} \\
& +\left\langle\left(c_{1}+\alpha_{1} I\right)\left(I-b_{1}\right) \varphi,\left(I-b_{1}\right) \varphi\right\rangle^{1 / 2}\left\langle\left(c_{2}+\alpha_{2} I\right) \psi, \psi\right\rangle^{1 / 2} \\
\leqq & \left\langle c_{1} \varphi ; \varphi\right\rangle^{1 / 2} 2 \alpha_{2}{ }^{1 / 2} \cdot\|\psi\|+2 \alpha_{1}{ }^{1 / 2}\|\varphi\|\left(\left\|c_{2}\right\|^{1 / 2}+\alpha_{2}{ }^{1 / 2}\right)^{i}\|\psi\|
\end{aligned}
$$

for all $\varphi, \psi \in \mathscr{H}$. This implies (3.4) 
Proof of Theorem 1 : First note that $\mathscr{B} \uparrow \mathscr{D} \subseteq \mathscr{L}$..Indeed, if $b \in \mathscr{B}$, then $B_{2}^{*} b B_{1 \mathrm{i}}$ $\in \mathscr{B}$ and so $b \uparrow \mathscr{D}=b_{2 \mathrm{j}}^{+} \circ\left(B_{2 \mathrm{j}}^{*} b B_{1 \mathrm{j}}\right) \circ b_{1 \mathrm{j}} \in \mathscr{L}$ for any' $\mathrm{j} \in \mathfrak{J}$. Fix an index $\mathrm{j} \in \mathfrak{J}$ and an operator $y \in \mathscr{B}$ and let $x=b_{2 i}^{+} \circ y \circ b_{1 j}$. It suffices to show that $x$ belongs to the closure of $\mathscr{B} \uparrow \mathscr{D}$ in $\mathscr{L}\left[\tau_{1 n}\right]$. For notational simplicity we omit the index $\mathrm{j}$ throughout the following proof. Take a positive number $\varepsilon$ satisfying $\varepsilon(1+\|y\|) \leqq 1$. Applying Lemma 2 in case $a=a_{k}, k=1,2$, we get for arbitrary $\varphi, \psi \in \mathscr{H}$.

$$
\begin{aligned}
\left|\left\langle\left(B_{2}^{2}\right)^{*} y B_{1}^{2} \varphi, \psi\right\rangle\right| & \leqq\|y\|^{2}\left\|B_{1}^{2} \varphi\right\|\left\|B_{2}^{2} \psi\right\| \\
& \leqq\|y\|^{2}\left(\varepsilon^{2}\|\varphi\|^{2}+\varepsilon^{-1}\left\|B_{1}^{3} \varphi\right\|^{2}\right)\left(\varepsilon^{2}\|\psi\|^{2}+\varepsilon^{-1}\left\|B_{2}{ }^{3} \psi\right\|^{2}\right) .
\end{aligned}
$$

That is, the assumptions of Lemma 3 are satisfied in case $z=\left(B_{2}{ }^{2}\right)^{*} y B_{1}{ }^{2}, \alpha_{k}=\varepsilon^{2}\|y\|$ and $c_{k}=\|y\| \varepsilon^{-1}\left(B_{k}^{3}\right)^{*} B_{k}^{3}$ for $k=1,2$. By Lemma 3 , there exist, operators $z_{1}, z_{2}$ and $\dot{y}_{1}$ in $\mathscr{B}$ such that $z=\left(B_{2}{ }^{2}\right)^{*} y B_{1}{ }^{2}=z_{1}+z_{2}, z_{1}=B_{2} y_{1} B_{1}$ and

$$
-\left\langle z_{2} \varphi, \psi\right\rangle \mid \leq \dot{\lambda} \varepsilon^{1 / 2}\|\varphi\|\|\psi\| \text { for } \varphi, \psi \in \dot{\mathscr{H}},
$$

where $\lambda$ is a certain constant depending only on the norms of $y, B_{1}$ and $B_{2}$. (We do not need the inequality (3.3) from Lemma 3.) Since $B_{1}$ and $B_{2}{ }^{*}$ are in $\mathscr{B}$, there is an $x_{1} \in \mathscr{B}$ such that $z_{1}=\left(B_{2}{ }^{3}\right)^{*}, x_{1} B_{1}{ }^{3}$. Define $x_{2}:=\left(b_{2}{ }^{+}\right)^{3} \circ z_{2} \circ b_{1}{ }^{3}$. Then

$$
\begin{aligned}
x & =\left(b_{2}{ }^{+}\right)^{3} \circ\left(\left(B_{2}{ }^{2}\right)^{*} y B_{1}{ }^{2}\right) \circ b_{1}{ }^{3}=\left(b_{2}{ }^{+}\right)^{3} \circ z_{1} \circ b_{1}{ }^{3}+\left(b_{2}{ }^{+}\right)^{3} \circ z_{2} \circ b_{1}{ }^{3} \\
& =\left(b_{2}{ }^{+}\right)^{3} \circ\left(\left(B_{2}{ }^{3}\right)^{*} x_{1} B_{1}{ }^{3}\right) \circ b_{1}{ }^{3}+x_{2}=x_{1} \uparrow \mathcal{D}+x_{2} .
\end{aligned}
$$

Therefore, from (3.7),

$$
\begin{aligned}
\left|\left\langle\left(x-\left(x_{1} \uparrow \mathscr{D}\right)\right) \varphi^{\prime}, \psi\right\rangle\right| & \equiv\left|\left\langle x_{2} \varphi, \psi\right\rangle\right|=\left|\left\langle z_{2} b_{1}{ }^{3} \varphi, b_{2}{ }^{3} \psi\right\rangle\right| \\
& \leq \lambda \varepsilon^{1 / 2}\left\|b_{1}{ }^{3} \varphi\right\|\left\|b_{2}{ }^{3} \psi\right\| \text { for all } \varphi, \psi \in \mathscr{H} .
\end{aligned}
$$

Since $x_{1} \in \mathscr{B}$ and $\lambda$ depends only on $y, B_{1}$ and $B_{2}$, this implies that $x$ is in the closure of $\mathscr{B} \uparrow \mathcal{D}$ in $\mathscr{L}\left[\tau_{\mathrm{in}}\right]$

\section{A generalization of Kaplansky's density theorem to spaces of sesquilinear forms}

We keep the assumptions and the notation from the beginning of Section 3 . Besides condition (I) from Section 3 , we need the following condition:

The family of seminorms $\|\cdot\|_{b_{k j}} \mathfrak{i} \in \mathfrak{J}$, is directed and generates the graph topo$\log y \mathrm{t}_{\mathcal{A}_{k}}$ on $D_{k}$ for $k=1,2$.

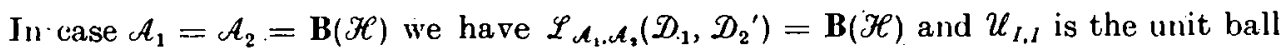
of $\mathbf{B}(\mathscr{H})$. Therefore, the following theorem can be considered as a generalization of the Kaplansky density theorem to some spaces of sesquilinear forms.

Theorem $1:$ Lel $\mathcal{A}_{1}$ and $\mathcal{A}_{2}$ be closed $O^{*}$-algebras on domains $\mathscr{D}_{1}$ and $\mathscr{D}_{2}$, respectively, of a Hilbert space $\mathscr{H}$ and let $\mathscr{B}$ be an $*$-subalgebra of $\mathbf{B}(\mathscr{H})$ : Assume that conditions (I) and (II) are satisfied. Let $\mathscr{L}$ be the linear span of $b_{2 \mathrm{i}}^{+} \circ \mathscr{B} \circ b_{1 \mathrm{i}}, \mathrm{i} \in \mathfrak{Y}$, and let $\mathscr{L}_{1}$ be another linear subspace of $\mathscr{L}_{\mathcal{A}_{1}, \mathcal{A}_{1}}\left(\mathscr{D}_{1}, \mathscr{D}_{2}{ }^{\prime}\right)$ which contains $\mathscr{L}$.

If $\mathscr{L}_{1}$ is in the weak-operator closure of $\mathscr{L}$ in $\mathscr{L}_{\mathcal{A}_{1}, \mathcal{A}_{2}}\left(\mathscr{D}_{1}, \mathscr{D}_{2}{ }^{\prime}\right)$, then $\mathscr{L} \cap \mathcal{U}_{b_{2 \mathrm{j}}, b_{1 \mathrm{j}}}$ is ultraweakly dense in $\dot{\mathscr{L}}_{1} \cap \mathcal{U}_{b_{2 \mathrm{j}}, b_{1 \mathrm{j}}}$ for each $\mathrm{j} \in \mathfrak{\Im}$.

Proof: Fix an index $\mathfrak{j} \in \mathfrak{\Im}$. Let $\mathfrak{F}_{\mathrm{j}}$ denote the closure of $\mathscr{B}$ in $\mathbf{B}(\mathscr{H})$ in the weakoperator topology with respect to $b_{1 \mathrm{j}} \mathscr{D} \times b_{2 \mathrm{j}} \mathscr{D}$. Clearly, $\mathscr{B}^{\prime \prime} \subseteq \mathfrak{F}_{\mathrm{j}}$. We show that $\mathfrak{S}_{\mathrm{i}}=\mathscr{B}^{\prime \prime}$. For let $x \in \mathfrak{C}_{\mathrm{j}}$. Then there is a net $\left\{x_{1}\right\}$ from $\mathscr{B}$ converging to $x$ in the weak- 
operator topology with respect to $b_{1 j} \mathscr{D} \times b_{2 \mathrm{j}} \mathcal{D}$. Suppose $y \in \mathscr{B}^{\prime}$ and $k \in\{1,2\}_{\text {. }}$ Since $B_{k j} \in \mathscr{B}$. by assumption, $y$ commutes with $B_{k \mathrm{i}}$ and herice with $\overline{b_{k j}}$ for each $j \in \mathfrak{I}$. Therefore, $\left.y \mathscr{D}_{k} \subseteq \bigcap\left\{D_{\left(b_{k j}\right)}\right) \mathrm{i} \in \Im\right\}$. Since $\mathcal{A}_{k}$ is assumed to be closed on $\mathscr{D}_{k}$, condition (II) implies that, the latter equals $\mathscr{D}_{k}$, so that $y \mathscr{D}_{k} \subseteq \mathscr{D}_{k}$. Because $\mathscr{B}^{\prime}$ is an *-algebra, $y^{*} \mathscr{D}_{k} \subseteq \mathscr{D}_{k}$. Therefore, if $\varphi \in \mathscr{D}_{1}$ and $\psi \in \mathscr{D}_{2}$, then $y b_{1 \mathrm{i}} \varphi=b_{1 \mathrm{i}} y \varphi \in b_{1 \mathrm{i}} \mathscr{D}_{1}$ and $y^{*} b_{2 \mathrm{i}} \psi=b_{2 \mathrm{i}} y^{*} \psi \in b_{2 \mathrm{i}} \mathscr{D}_{2}$ and hence

$$
\begin{aligned}
\left\langle x y b_{1 \mathrm{i}} \varphi, b_{2 \mathrm{i}} \psi\right\rangle & =\lim _{\mathfrak{l}_{1}}\left\langle x_{1} y b_{1 \mathrm{i}} \varphi, b_{2 \mathrm{i}} \psi\right\rangle=\lim _{\mathfrak{l}}\left\langle x_{1} b_{1 \mathrm{i}} \varphi ; y^{*} b_{\mathrm{ij}} \psi\right\rangle \\
= & \left\langle x b_{1 \mathrm{i}} \varphi, y^{*} b_{2 \mathrm{i}} \psi\right\rangle=\left\langle x y \bar{b}_{1 \mathrm{i}} \varphi, b_{2 \mathrm{i}} \psi\right\rangle
\end{aligned}
$$

Since $b_{1 \mathrm{i}} \mathscr{D}_{1}$ and $b_{2 \mathrm{i}} \mathscr{D}_{2}$ are dense in $\mathscr{H}$ by $(\mathrm{I})$, this yields $x y=y x$. Thus $x \in \mathscr{B}^{\prime \prime}$ and $\mathfrak{\aleph}_{\mathrm{j}}=\mathscr{B}^{\prime \prime}$.

By Lemma 1.1, for each $\bar{x} \in\left(\mathscr{L}_{1}\right)_{b_{2 j}, b_{1 j}}$ there is an operator $y \in \mathbf{B}(\mathscr{H})$. such that $x=b_{2 \mathrm{j}}^{+} \circ y \circ b_{1 \mathrm{j}}$. Let $\mathscr{B}_{\mathrm{j}}$ denote the set of all such operators $y$ if $x$ runs through $\left(\mathscr{L}_{1}\right)_{b_{2 \mathrm{j}} \cdot b_{1 \mathrm{i}}}$. Since $b_{k \mathrm{i}} \mathscr{D}_{k}$ is dense in $\mathscr{H}$ for $k=1,2, b_{2 \mathrm{j}}^{+} \circ y_{1} \circ b_{1 \mathrm{i}}=b_{2 \mathrm{j}}^{+} \circ \dot{y}_{2} \circ b_{1 \mathrm{i}}$ for $y_{1}, \dot{y_{2}} \in \mathbf{B}(\mathscr{H})$ implies that $y_{1}=y_{2}$. From $\dot{b}_{2}^{+} \circ \mathscr{B} \circ b_{1 \mathrm{i}} \cong \mathscr{L}_{b_{2 \mathrm{j}}, b_{1 \mathrm{j}}} \cong\left(\mathscr{L}_{1}\right)_{b_{2 \mathrm{j}}, b_{1 \mathrm{j}}}$ $=b_{2 \mathrm{j}}^{+} \circ \mathscr{B}_{\mathrm{j}} \circ b_{1 \mathrm{j}}$ we therefore conclude that $\mathscr{B} \subseteq \dot{\mathscr{B}}_{\mathrm{j}}$.

We prove that $\mathscr{B}_{\mathrm{i}} \subseteq \mathscr{B}^{\prime \prime}$. By Theorem 1 in Section $3, \mathscr{B} \uparrow \mathscr{D}$ is in $\mathscr{L}\left[\tau_{\mathrm{in}}\right]$ and hence; of course, dense in $\mathscr{L}$ in the weak-operator topology. Since $\mathscr{L}$ is weak-operator dense in $\mathscr{L}_{1}$ by assumption, $\mathscr{B} \uparrow \mathscr{D}$ is weak-operator dense in $\mathscr{L}_{1}$. Suppose $\mid y \in \mathscr{B}_{\mathrm{i}}$. Theri $b_{2 j} \circ y \circ b_{1 \mathrm{i}} \in \mathscr{L}_{1}$, so that there exists a net $\left\{x_{1} \uparrow \mathcal{D}\right\}$ from $\mathscr{B} \uparrow \mathcal{D}$ which converges to $b_{2 \mathrm{i}} \circ y \circ b_{1 \mathrm{i}}$ in the weak-operator topology with respect to $\mathscr{D}_{1} \times \mathscr{D}_{2}$. Let $\varphi \in b_{1 \mathrm{i}} \mathscr{D}_{1}$ and $\psi \in b_{2 \mathrm{j}} \mathscr{D}_{2}$. Then $B_{1 \mathrm{i}} \varphi \in \mathscr{D}_{1}$ and $B_{2 \mathrm{j}} \psi \in \mathscr{D}_{2}$ and hence

$$
\begin{aligned}
\lim _{1}\left\langle x_{1} B_{\mathrm{ii}} \varphi, B_{2 \mathrm{i}} \psi\right\rangle & =\lim _{.1}\left\langle B_{2 \mathrm{i}}^{*} x_{\mathrm{l}} B_{1 \mathrm{i}} \varphi, \psi\right\rangle \\
& =\left\langle\left(b_{2 \mathrm{i}}^{+} \circ y, \circ b_{1 \mathrm{i}}\right) B_{1 \mathrm{i}} \varphi, B_{2 \mathrm{i}} \psi\right\rangle=\langle y \varphi, \dot{\psi}\rangle
\end{aligned}
$$

Since $B_{2 \mathrm{j}}^{*} x_{\mathrm{l}} B_{1 \mathrm{i}} \in \mathscr{B}$ for all $\mathfrak{l}$, this shows that $y \in \mathfrak{E}_{\mathrm{j}}$ : Because $\mathfrak{C}_{\mathrm{j}}=\mathscr{B}^{\prime \prime}$ as shown above, we have $y \in \mathscr{B}^{\prime \prime}$. Thus $\mathscr{B}_{\mathrm{i}} \subseteq \mathscr{B}^{\prime \prime}$.

Let $\mathscr{\mathscr { B }}_{\mathrm{i}}$ denote the $*$-subalgebra of $\mathbf{B}(\mathscr{H})$ which is generated by $\mathscr{B}_{\mathrm{j}}$. Since $\mathscr{B}_{\mathrm{i}} \subseteq \mathscr{B}^{\prime \prime}$, $\mathscr{B} \subseteq \tilde{\mathscr{B}}_{\mathrm{i}} \leqq \mathscr{B}^{\prime \prime}$. That is, the *-algebra $\mathscr{B}$ is dense in the *-algebra $\tilde{\mathscr{B}}_{\mathrm{i}}$ in the weakoperator topology of $\mathbf{B}(\mathscr{H})$. Let $\mathcal{U}_{1}$ be the unit ball of $\mathbf{B}(\mathscr{H})$. Kaplansky's density theorem (see e.g. $[8$, p. 329$]$ ) states that $\mathscr{B} \cap U_{1}$ is ultraweakly dense in $\widetilde{\mathscr{B}}_{\mathfrak{j}} \cap \mathscr{U}_{1}$ and so in $\mathscr{B}_{i} \cap U_{1}$. This implies that the subset $b_{2 \mathrm{i}}^{+} \circ\left(\mathscr{B}_{\cap} \cap U_{1}\right) \circ b_{1 \mathrm{i}}$ of $\mathscr{L}_{1} \cap \mathscr{L}_{b_{2 i}, b_{1 \mathrm{i}}}$ is ultraweakly dense in $b_{2 \mathrm{i}}^{+} \circ\left(\mathscr{B}_{\mathrm{i}} \cap U_{1}\right) \circ b_{1 \mathrm{i}}$. Since $\dot{b}_{2 \mathrm{i}}^{+} \circ\left(\dot{\mathscr{B}}_{\mathrm{j}} \cap U_{1}\right) \circ b_{1 \mathrm{i}}=\mathscr{L}_{1} \cap U_{b_{2 \mathrm{i}} \cdot \dot{b}_{1 \mathrm{i}}}$ by the density of $b_{1 \mathrm{i}} \mathscr{D}_{1}$ and $b_{2 \mathrm{i}} \mathscr{D}_{2}$ in $\mathscr{H}$, this proves the assertion

A by-product of the preceding proof is

Corollary 2: Let $\mathcal{A}_{1}, \mathcal{A}_{2}, \dot{B}, \mathscr{L}$ and $\left\{b_{k \mathrm{j}} ; \mathrm{i} \in \mathfrak{S}\right\}, \dot{k}=1,2$, be as in Theorem 1'. Then the closures of $\mathscr{L}$ in the weak-operator topology and in the ultraweak topology within $\mathscr{L}_{\mathcal{A}_{1}, \mathcal{C}_{2}}\left(\mathscr{D}_{1}, \mathscr{D}_{2}^{\prime}\right)^{\prime}$ coincide and they are equal to $\bigcup_{\mathrm{i} \in \mathcal{\Im}} b_{2 \mathrm{j}}^{+} \circ \mathscr{B}^{\prime \prime} \circ b_{1 \mathrm{i}}$.

-Proof: Let $\mathscr{L}_{1}$ denote the weak-operator closure of $\mathscr{L}_{0}:=\bigcup_{\mathrm{i} \in \Im} b_{2 \mathrm{i}}^{+} \circ \mathscr{B}^{\prime \prime} \circ b_{1 \mathrm{i}}$ within $\mathscr{L}_{\mathcal{A}_{1}, \mathcal{A}_{1}}\left(\mathscr{D}_{1}, \mathscr{D}_{2}^{\prime}\right)$ and let $\left(\mathscr{B}^{\prime \prime}\right)_{\mathrm{i}}, \mathfrak{j} \in \mathfrak{I}$, be the corresponding subsets for $\mathscr{L}_{1}$ as defined in the proof of Theorem 1 . The proof of Theorem 1 (with $\mathscr{L}$ and $\mathscr{B}$ replaced by $\mathscr{L}_{0}$ and $\mathscr{B}^{\prime \prime}$, respectively) showed that $\mathscr{B}^{\prime \prime} \subseteq\left(\mathscr{B}^{\prime \prime}\right)_{\mathrm{i}} \subseteq\left(\mathscr{B}^{\prime \prime}\right)^{\prime \prime}$. That is, $\mathscr{B}^{\prime \prime}=\left(\mathscr{B}^{\prime \prime}\right)_{\mathrm{i}}$ for $\mathrm{i} \in \mathfrak{\Im}$. Thus $\mathscr{L}_{0}=\mathscr{L}_{1}$, so that $\mathscr{L}_{0}$ is weak-operator and hence ultraweakly closed in 
$\mathscr{L}_{\mathcal{A}_{1}, \mathcal{A}_{z}}\left(\mathscr{D}_{1}, \mathscr{D}_{2}{ }^{\prime}\right)$. On the other hand, by the von Neumann density theorem; $\mathscr{B}$ is ultraweakly dense in $\mathscr{B}^{\prime \prime}$.'This implies that $\mathscr{L}$ is ultraweakly and hence weak-operator dense in $\mathscr{L}_{0}$. Combined with the preceding, the assertion follows

An immediate consequence of Corollary 2 is

Corollary 3:, Under the assumptions and notations of Theorem 1, the following three conditions are equivalent:

(i) $\mathscr{L}$ is weak-operator closed in $\mathscr{L}_{\mathcal{A}_{1}, \mathcal{A}_{2}}\left(\mathscr{D}_{1}, \mathscr{D}_{2}{ }^{\prime}\right)$.

(ii) . $\mathscr{L}$ is ultraweakly closed in $\mathscr{L}_{\mathcal{A}_{1}, \mathcal{A}_{2}}\left(\mathscr{D}_{1}, \mathscr{D}_{2}{ }^{\prime}\right)$.

(iii) $\mathscr{B}$ is a von Neumann algebra.

In case where $\mathcal{A}_{2}=\mathbf{B}(\mathscr{H})$ and $\mathscr{D}_{2}=\ddot{\mathscr{H}}$ we have similar assertions for the ultrastrong topology.

Theorem'4: Suppose $\mathcal{A}_{1}$ is a closed $O^{*}$-algebra on $\mathscr{D}_{1}$ such that (I) and (II) are fulfilled in case $k=1$. Let $\mathscr{L}$ be the vector space of operators on $\mathscr{D}_{1}$ generated by $x b_{1}$, where $x \in \mathscr{B}$ and $\mathrm{i} \in \mathfrak{S}$. (Here the set $\left\{b_{1 \mathrm{i}} ; \mathrm{j} \in \mathfrak{S}\right\}$ and the $*$-subalgebra $\mathscr{B}$ are as in (I) and (II) for $k=1$.) Let $\mathscr{L}_{1}$ be a linear subspace of $\mathscr{L}_{\mathcal{A}_{3}}\left(\mathscr{D}_{1}, \mathscr{H}\right)$ such that $\mathscr{L} \subseteq \mathscr{L}_{1}$.

If $\mathscr{L}$ is dense in $\mathscr{L}_{1}$ in the weak-operator topology with respect to $\mathscr{D}_{1} \times \mathscr{H}$, then, for each $\mathrm{i} \in \mathfrak{J}, \mathscr{L} \cdot \cap \mathcal{U}_{b_{1 \mathrm{i}}}$ is dense in $\mathscr{L}_{1} \cap \mathcal{U}_{b_{1 \mathrm{i}}}$ in the ultrastrong topology.

Proof: The proof is very similar to the proof of Theorem $i$ in case $\mathcal{A}_{2}=\mathbf{B}(\mathscr{H})$, $\mathscr{D}_{2}=\mathscr{H}$. At the end of this proof it suffices to replace the ultraweak density of $\mathscr{B}^{\circ} \cap U_{1}$ in $\widehat{\mathscr{B}}_{\mathrm{i}} \cap U_{1}$ by the ultrastrong density which follows also from the Kaplansky density theorem for von Neumann àlgebras

Assume that $\mathfrak{A}_{1}, \mathscr{B}, \mathscr{L}$ and $\left\{b_{1 \mathrm{j}} ; \mathrm{i} \in \mathfrak{\}}\right\}$ are as in Theorem 1. Then the following two corollaries can be derived in a similar way as Corollaries 2 and 3 above.

Corollary 5: The closures of $\mathscr{L}$ with respect to the weak-operator topology, the ultraweak topology (both with respect to $\mathcal{D}_{1} \times \mathscr{H}$ ), the strong-operator topology and the ultrastrong topology in $\mathscr{L}_{\mathcal{A}_{1}}\left(\mathscr{D}_{1}, \mathscr{H}\right)$ 'coincide. They are equal to $\cup \mathscr{B}^{\prime \prime} \cdot b_{1 \mathrm{i}}$.

Corollary 6: The following three conditions are equivalent:

(i) $\quad \mathscr{L}$ is weak-operator closed (with respect to $\mathscr{D}_{1} \times \mathscr{H}$ ) in $\mathscr{L}_{\mathcal{A}_{1}}\left(\mathscr{D}_{1}, \mathscr{H}\right)$.

(ii) $\quad \mathscr{L}$ is ultrastrongly closed in $\mathscr{L}_{\mathcal{M}_{1}}\left(\mathscr{D}_{1}, \mathscr{H}\right)$.

(iii) $\quad \mathscr{B}$ is a von Neumann algebra.

Acknowledgment. This work was done in Fall 1985 while the author was visiting the University of Iowa in Iowa City and the University of Pennsylvania in Philadelphia. He would like to thank Professors J. Cuntz, P. E. T. Jørgensen, R. V. Kadison, P. S. Muhly and R. T. Powers for their hospitality.

\section{REFERENCES}

[1] Araki, H., and J. P. Jurzak: On a certain class of -algebras of unbounded operators. Publ. Res. Inst. Math. Sci. (RIMS) Kyoto Univ. 18 (1982), 1013-1044.

[2] ArNaL, D., and J. P. JuRzak: Topological aspects of algebras of unbounded operators. J. Funct. Anal. 24 (1977), 397-425.

[3] Borchers, H. J., and J. Yxavason: On the algebra of field operators. The weak commutant and integral decomposition of states. Commun. Math. Phys. 42 (1975), 231-252.

[4] Dixon, P. G.: Unbounded operator algebras. Proc. London Math. Soc. 23 (1971), 53-69. 
[5] GUDDER, S. P., and R. L. Hudson: A noncommutative probability theory. Trans. Amer. Math. Soc. $\mathbf{9 4 5}$ (1978), $1-41$.

[6] Inove, A.: A class of unbounded operator algebras. Pacific J. Math. 65 (1976), 77-96.

[7] Jurzak, J. P.: Unbounded Non-Commutative Integration. Dordrecht: D. Reidel. Publ. Comp. 1986.

[8] Kadison, R. V., and J. R. Ringrose: Fundamentals of the theory of operator algebras, Vol. I: New York: Acad. Press 1983.

[9] Kröger, P.: On EC*-algebras. Preprint. Leipzig: Karl-Márx-Universität 1978.

[10] Kürstes, K. D.: The completion of the maximal Op*algebra on a Frechet domain. Publ. Res. Inst. Math. Sci. (RIMS) Kyoto Univ. 22 (1986), 151-175.

- [11] Kürstex, K. D.: Duality for maximal Op*-algebras on Frechet domains. Preprint. Leipzig: Karl-Marx-Universität 1986 (to appear).

[12] Lassner, G.: Topological algebras of operators. Rep. Math. Phys. 3 (1972), 279-293.

[13] LaSSNER, G.: Quasi-uniform topologies on local observables. Acta Univ. IVraticlaviensis $519(1979), 43-60$.

[14] Matrot, F.: Topological properties of unbounded bicommutants. J. Math. Phys. 26 $(1985), 1118-1124$.

[15] Powers, R. T.: Self-adjoint algebras of unbounded operators. Commun. Math. Phys. 21 (1971), 85-124.

[16] SchmüDGex, K.: Two theorems about topologies on countably generated Op*-algebras. Acta Math. Sci. Acad. Hungar. 35 (1980), 139-150.

[17] TAKESAKI, M.: Theory of Operator Algebras I. Berlin-Heidelberg-New York : SpringerVerlag. 1979.

- Manuskripteingang: 08. 09.1987

VERFASSER:

Prof. Dr. Konrad Schiüdaen

Sektion Mathematik der Karl-Marx-Universität

Karl-Marx-Platz 10

DDR - 7010 Leipzig 